\title{
Potencial de Lixiviação de Herbicidas no Solo SubMetidos a Diferentes Simulações de Precipitação ${ }^{1}$
}

\author{
Leaching Potential of Herbicides in Soil under Different Rainfall Simulations
}

MONQUERO, P.A. ${ }^{2}$, AMARAL, L.R. ${ }^{3}$, BINHA, D.P. ${ }^{5}$, SILVA, A.C. ${ }^{4}$ e SILVA, P.V. ${ }^{5}$

\begin{abstract}
RESUMO - O objetivo deste trabalho foi avaliar a influência da precipitação pluvial na lixiviação de herbicidas recomendados para a cultura da cana-de-açúcar em Latossolo Vermelho distrófico. O experimento consistiu da aplicação de trifloxysulfuron-sodium + ametryn $\left(1.463+37 \mathrm{~g} \mathrm{ha}^{-1}\right)$, imazapic $\left(84 \mathrm{~g} \mathrm{ha}^{-1}\right)$, imazapyr $\left(200 \mathrm{~g} \mathrm{ha}^{-1}\right),{ }^{1}$ diuron + hexazinone $\left(1.170+330 \mathrm{~g} \mathrm{ha}^{-1}\right)$ (formulação 1$) \mathrm{e}^{2}$ diuron + hexazinone $\left(1.330+160 \mathrm{~g}\right.$ ha ${ }^{1}$ ) (formulação 2) no topo de colunas de solo montadas em tubos de PVC. Foram simuladas precipitações pluviais de 20, 40 e $80 \mathrm{~mm}$ após a aplicação dos herbicidas. As avaliações foram realizadas aos 14 e 21 dias após a aplicação dos tratamentos (DAT). Os tubos de PVC foram desmontados para a semeadura do bioindicador Cucumis sativus ao longo das colunas. Após $20 \mathrm{~mm}$ de chuva, o herbicida trifloxysulfurom-sodium + ametryn provocou sintomas decrescentes de fitotoxicidade nas plântulas que emergiram de 0 a $20 \mathrm{~cm}$; já os demais herbicidas causaram efeito até $10 \mathrm{~cm}$. A precipitação de $40 \mathrm{~mm}$ fez com que as misturas ${ }^{1,2}$ diuron + hexazinone provocassem injúrias severas nas plantas de pepino até $15 \mathrm{~cm}$ de profundidade e sintomas decrescentes até a profundidade de $20 \mathrm{~cm}$ (formulação 2) e $25 \mathrm{~cm}$ (formulação 1). Os herbicidas trifloxysulfurom-sodium + ametryn e imazapyr foram detectados até $20 \mathrm{~cm}$ de profundidade. O herbicida imazapic causou fitotoxicidade até $15 \mathrm{~cm}$ de profundidade. Com $80 \mathrm{~mm}$ de chuva, o herbicida ${ }^{1}$ diuron + hexazinone causou fitotoxicidade até $35 \mathrm{~cm}$, e os herbicidas ${ }^{2}$ diuron + hexazinone, trifloxysulfurom-sodium + ametryn, imazapyr e imazapic, até $25 \mathrm{~cm}$. Concluiu-se que todos os herbicidas avaliados têm tendência a serem lixiviados por influência das precipitações pluviais ou de irrigações artificiais.
\end{abstract}

Palavras-chave: cana-de-açúcar, chuva, bioensaio, herbicidas.

\begin{abstract}
The objective of this work was to evaluate the influence of rainfall on leaching of herbicides recommended for sugarcane crop in Dystrophic Red Latosol. The experiment consisted in spraying trifloxysulfuron-sodium + ametryn $\left(1463+37 \mathrm{~g} \mathrm{ha}^{-1}\right)$, imazapic $\left(84 \mathrm{~g} \mathrm{ha} \mathrm{h}^{-1}\right)$, imazapyr $\left(200 \mathrm{~g} \mathrm{ha}^{-1}\right)$, ${ }^{1}$ diuron + hexazinone $\left(1170+330 \mathrm{~g} \mathrm{ha}^{-1}\right)$ and ${ }^{2}$ diuron + hexazinone $\left(1330+160 \mathrm{~g} \mathrm{ha}^{-1}\right)$ on top of PVC columns filled with soil. After spraying, simulated rainfall of 20,40 and $80 \mathrm{~mm}$ was applied on top of the columns. Cucumis sativus (cucumber) was used in bioassays to detect herbicide leaching, with evaluations accomplished 14 and 21 days after application of the treatments. After a $20 \mathrm{~mm}$ rainfall simulation, it was verified that the trifloxysulfuron-sodim + ametrin mixtures caused decreasing symptoms of toxicity in the 0 to $20 \mathrm{~cm}$ plantules, while the other herbicides had an effect up to $10 \mathrm{~cm}$. The $40 \mathrm{~mm}$ rainfall made the mixtures $\mathrm{s}^{1,2}$ diuron + hexazinone cause severe symptoms in the cucumber plants at $15 \mathrm{~cm}$ depth and decreasing symptoms at depths of $20 \mathrm{~cm}$ (formulation 2) and $25 \mathrm{~cm}$ (formulation 1). The herbicides trifloxysulfurom sodium + ametryn and imazapyr were detected up to $20 \mathrm{~cm}$ of depth. The herbicide imazapic caused toxicity up to $15 \mathrm{~cm}$ of depth. At $80 \mathrm{~mm}$ rainfall, ${ }^{1}$ diuron + hexazinone caused toxicity up
\end{abstract}

Recebido para publicação em 10.9.2007 e na forma revisada em 31.3.2008.

2 Professora adjunta do Centro de Ciências Agrárias/UFSCar, Rodovia Anhanguera, km 174, 13600-970, Araras, SP, <pamonque@cca.ufscar.br>; ${ }^{3}$ Aluno de graduação do Centro de Ciências Agrárias/UFSCar - bolsista da Fapesp; ${ }^{4}$ Pesquisadora científica do Pólo Regional da Alta Sorocabana, Rodovia Raposo Tavares, km 561 Caixa Postal 298, 19015-970 Presidente Prudente-SP; 5 Alunos de graduação do Centro de Ciências Agrárias/UFSCar. 
to $30 \mathrm{~cm}$ and ${ }^{2}$ diuron + hexazinone, imazapyr, trifloxysulfuron sodium + ametryn and imazapic presented toxicity up to $25 \mathrm{~cm}$ of depth. It was concluded that all the herbicides evaluated tend to be leached under the influence of rainfall or artificial irrigations.

Keywords: sugarcane, rainfall, bioassay, herbicides.

\section{INTRODUÇÃO}

No cultivo de grandes áreas, atualmente ocorre utilização intensiva de insumos, com os defensivos figurando entre os mais importantes para obtenção de alto indice de produtividade. Por outro lado, o uso exagerado e inadequado dessas moléculas organossintéticas pode acarretar sérias conseqüências para o meio ambiente (Barizon et al., 2006).

Devido ao seu uso intensivo, os herbicidas são freqüentemente detectados em estudos de qualidade de águas superficiais e subterrâneas (Carter, 2000; Tanabe et al., 2001). Nas áreas próximas ao cultivo de cana-de-açúcar registra-se maior ocorrência de resíduos desses compostos, já que esta é uma das culturas que mais utilizam herbicidas no manejo de plantas infestantes ou plantas daninhas (Southwick et al., 2002; Vivian et al., 2007).

No solo, quando o herbicida é aplicado em pré-emergência, pode sofrer processos de sorção, lixiviação e/ou degradação por efeitos físicos, químicos e biológicos, além de ser absorvido pelas plantas daninhas e/ou plantas cultivadas (Velini, 1992).

O processo de lixiviação é a principal forma de transporte no solo das moléculas não-voláteis e solúveis em água. Essas moléculas se deslocam no perfil do solo, acompanhando o fluxo de água, o qual é governado pela diferença de potencial de água entre dois pontos (Prata et al., 2003). A lixiviação é fundamental para a incorporação superficial da maioria dos herbicidas, atingindo sementes ou plantas em germinação, mas, quando excessiva, pode carreálos para camadas mais profundas do solo, limitando sua ação e podendo, inclusive, promover contaminação do lençol freático (Velini, 1992).

Esses processos são dependentes do tipo de solo, das condições climáticas e das características dos produtos. O conhecimento desses fatores é de fundamental importância para prever o comportamento de herbicidas nas diferentes classes de solo e para seleção de dosagens adequadas, bem como para evitar efeitos prejudiciais ao ambiente e às culturas subseqüentes (Rossi et al., 2005).

O movimento descendente dos herbicidas no solo é influenciado pelo teor e tipo de matéria orgânica, composição, tamanho e distribuição das partículas do solo, $\mathrm{pH}$ e densidade do solo, tamanho e distribuição dos poros, além da solubilidade em água das moléculas dos herbicidas e do indice de precipitação pluvial (Prata et al., 2003). Para herbicidas de elevada capacidade adsortiva, o conteúdo de matéria orgânica do solo é geralmente um fator importante, pois, quanto mais alto, maior será a adsorção do herbicida e, com isso, menor a lixiviação. Em solos arenosos, a lixiviação será ainda maior do que em solos siltosos ou argilosos (Rossi et al., 2005).

Em solos com baixo teor de matéria orgânica e CTC efetiva, verificou-se, por exemplo, aumento na ocorrência de efeitos fitotóxicos do s-metolachlor às culturas e na probabilidade de contaminação de águas subterrâneas (Procópio et al., 2001).

No Mato Grosso, foram detectados resíduos de ametryn na maioria das amostras coletadas em águas de superficie, embora em baixa concentração. Entretanto, em amostras de sedimentos, as concentrações foram superiores a $4,5 \mu \mathrm{g} \mathrm{kg}^{-1}$. A elevada freqüência e concentração de ametrina nessas amostras foi atribuída ao intenso cultivo de cana-de-açúcar no nordeste do pantanal (Laabs et al., 2002).

Simulação de aplicação dos herbicidas atrazine, diuron e tebuthiuron realizada por Pessoa et al. (1999) e Matallo et al. (2003) em áreas de recarga do Aqüífero Guarani revelou que todos os herbicidas se deslocam mais no 
perfil do Neossolo Quartazênico Órtico do que no Latossolo Vermelho Psamítico, com destaque para o tebuthiuron, que atingiu 9,43 m num período simulado de quatro anos.

O monitoramento de herbicidas realizado entre 1992 e 2001 no sul da Flórida revelou que ametryne e atrazine foram os compostos mais comumente encontrados em águas superficiais (Pfeuffer \& Rand, 2004). Mitchell et al. (2005), citados por Vivian et al. (2007), identificaram, em solo cultivado com cana-deaçúcar, resíduos de ametryn $\left(0,3 \mu \mathrm{g} \mathrm{\textrm {L } ^ { - 1 } )}\right.$ e mais outros quatro herbicidas em águas de rios da Austrália.

No estudo do movimento da molécula de herbicidas em solos, pesquisadores têm utilizado o método do bioensaio, rápido e de baixo custo. Essa técnica consiste na utilização de plantas sensiveis aos produtos testados, de forma que residuos de herbicidas presentes no solo possam ser evidenciados por meio da apresentação de sintomas de injúrias na planta-teste (Pasqualetto et al., 1999).

Em geral, pode-se esperar correlação positiva entre a precipitação pluvial e a lixiviação de herbicidas, e as precipitações intensas podem incrementar a lixiviação desses produtos e contaminar as águas subsuperficiais.

O objetivo deste trabalho foi estudar a lixiviação dos herbicidas trifloxysulfuron-sodium + ametryn, imazapic, imazapyr e diuron + hexazinone em diferentes simulações de precipitações pluviais.

\section{MATERIAL E MÉTODOS}

O experimento foi conduzido durante o período de abril a maio de 2007, em casa de vegetação, no Centro de Ciências Agrárias/UFSCar, Araras-SP. As amostras de solos foram retiradas de um solo classificado como Latossolo Vermelho-Escuro (Embrapa, 1999), nas profundidades de 0-20, 20-35 e 35-50 cm. Estas amostras foram secas, moídas e peneiradas em malha de $2 \mathrm{~mm}$. A análise química revelou $\mathrm{pH}$ de 5,3 em $\mathrm{CaCl}_{2} \mathrm{~g} \mathrm{dm}^{-3}$ de matéria orgânica, $12 \mathrm{mg} \mathrm{dm}^{-3}$ de $\mathrm{P}$ resina, $63 \%$ de $\mathrm{V}$ e teores de $\mathrm{K}, \mathrm{Ca}, \mathrm{Mg}, \mathrm{H}+\mathrm{Al}$ e $\mathrm{SB}$ de 2,$3 ; 28 ; 11 ; 24$; $41,3 \mathrm{mmol}_{\mathrm{c}} \mathrm{dm}^{-3}$, respectivamente.

As unidades experimentais constaram de colunas de solo montadas em tubos de PVC de
$10 \mathrm{~cm}$ de diâmetro e $50 \mathrm{~cm}$ de comprimento. Os tubos foram envoltos internamente por uma camada de parafina, a fim de evitar escorrimento lateral da solução do solo. Na parte basal, para reter o solo e permitir a drenagem, foram colocadas telas de sombrite $50 \%$. As colunas de solo foram submetidas à irrigação por capilaridade, até este se apresentar saturado no topo, permitindo-se em seguida o escoamento da água durante 48 horas, para restaurar sua capacidade de campo.

O ensaio constituiu na aplicação dos herbicidas trifloxysulfuron-sodium + ametryn

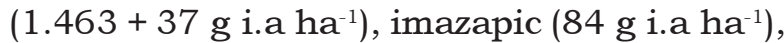
imazapyr (200 g i.a ha-1), ${ }^{1}$ diuron + hexazinone $\left(1.170+330 \mathrm{~g}^{\text {i.a ha }} \mathrm{h}^{-1}\right)$ (formulação 1$) \mathrm{e}^{2}$ diuron + hexazinone $\left(1.330+160 \mathrm{~g}^{\text {i.a }} \mathrm{ha}^{-1}\right)$ (formulação 2). Os tratamentos foram arranjados em esquema fatorial $5 \times 8$, sendo cinco herbicidas e oito profundidades, avaliados dentro de cada precipitação.

Os herbicidas foram aplicados, separadamente, com pulverizador costal pressurizado com $\mathrm{CO}_{2}$, provido de barra de pulverização contendo dois bicos tipo leque Teejet 110.02, com volume de aplicação de $200 \mathrm{~L} \mathrm{ha}^{-1}$.

Logo após a aplicação dos herbicidas, os tubos foram colocados sob simulador de chuva, com intensidade de precipitação de $1 \mathrm{~mm} \mathrm{~min}^{-1}$, durante o tempo necessário para aplicar as lâminas de 0, 20, 40 e $80 \mathrm{~mm}$. Após a simulação, as colunas ficaram em repouso por 72 horas, quando então foram abertas e colocadas na posição horizontal. Cada coluna foi dividida em oito seções de $5 \mathrm{~cm}$, medidas a partir da superficie onde os herbicidas foram aplicados. Em cada seção foi semeada uma linha contínua de sementes de pepino Cucumis sativus, cuja planta é indicadora de injúrias e sensivel aos herbicidas.

Aos 14 e 21 dias após a semeadura, foram observadas visualmente possiveis alterações nas plântulas de pepino que pudessem ser caracterizadas como efeitos tóxicos dos produtos. Essas avaliações foram baseadas em critérios qualitativos, segundo a ALAM (1974), que utiliza uma escala percentual de notas, em que 0 corresponde a nenhuma injúria na planta e 100 , à morte das plantas.

Os dados obtidos foram submetidos à análise de variância, sendo plotadas curvas 
de regressão utilizando o programa SIGMA PLOT.

\section{RESULTADOS E DISCUSSÃO}

Houve interação significativa entre os herbicidas avaliados e as diferentes profundidades dentro de cada regime pluvial avaliado, indicando que os herbicidas diferiram em relação à mobilidade no perfil das colunas.

Os sintomas observados aos 14 e 21 DAT devido aos herbicidas trifloxysulfurom-sodium + ametryn, imazapic e imazapyr foram a paralisação do desenvolvimento aéreo e início de encarquilhamento das folhas. Já os herbicidas ${ }_{1,2}$ diuron + hexazinone promoveram amarelecimento mais evidente e necrose das folhas do bioindicador.

Quando não houve simulação de chuva, todos os herbicidas ficaram retidos na superficie do solo, sem se detectar lixiviação, e apresentando menor disponibilidade, o que pode ser observado pelo efeito insatisfatório dos herbicidas nas plantas bioindicadoras aos 14 e 21 DAT (Figuras 1 e 2). Com a simulação de $20 \mathrm{~mm}$ de chuva, o herbicida trifloxysulfurom-sodium + ametryn provocou sintomas decrescentes de fitotoxicidade nas plântulas que emergiram de 0 a $20 \mathrm{~cm}$; já os demais herbicidas causaram efeito até $10 \mathrm{~cm}$ de profundidade (Figuras $1 \mathrm{e}$ 2). É importante lembrar que em áreas cultivadas, nos primeiros $20 \mathrm{~cm}$ do perfil do solo, podem-se encontrar $90 \%$ das sementes de plantas daninhas (Buhler et al., 1997).

Na Figura 2, observa-se que a precipitação de $40 \mathrm{~mm}$ fez com que as misturas ${ }^{1,2}$ diuron + hexazinone (formulações 1 e 2) aos 21 DAT provocassem injúrias muito severas nas plantas de pepino até $15 \mathrm{~cm}$ de profundidade, com $100 \%$ de controle e sintomas decrescentes até a profundidade de $20 \mathrm{~cm}$ (formulação 2) e $25 \mathrm{~cm}$ (formulação 1). Os herbicidas trifloxysulfuromsodium + ametryn e imazapyr foram detectados pelo bioensaio até $20 \mathrm{~cm}$ de profundidade, porém com controle menor do bioindicador $(80 \%)$, comparando-se ao diuron + hexazinone, e com injúrias leves na profundidade de $25 \mathrm{~cm}$ com $20 \%$ de controle. Já o herbicida imazapic causou fitotoxicidade até $15 \mathrm{~cm}$ de profundidade. Inoue et al. (2006) verificaram que, com $40 \mathrm{~mm}$ de água, a lixiviação do imazapic (65 e
$130 \mathrm{~g} \mathrm{ha}^{-1}$ ) ocorreu até a camada de 10-15 ou de $15-20 \mathrm{~cm}$, dependendo da dose e do bioindicador (Brachiaria decumbens ou Cucumis sativus).

Com 80 mm de chuva, o herbicida ${ }^{1}$ diuron + hexazinone causou fitotoxicidade até $35 \mathrm{~cm}$, e o ${ }^{2}$ diuron + hexazinone, até $25 \mathrm{~cm}$. A formulação 1 ( ${ }^{1}$ diuron + hexazinone) apresenta concentração maior de hexazinone, que é um ingrediente ativo de alta solubilidade $(29.800 \mathrm{ppm}$ a $25{ }^{\circ} \mathrm{C}$ ), necessitando de menos umidade no solo para se movimentar, quando comparado ao diuron $\left(42 \mathrm{ppm}\right.$ a $\left.25^{\circ} \mathrm{C}\right)$ (Bouchard et al., 1985).

A lixiviação de defensivos agrícolas no perfil do solo tem implicações diretas na contaminação de recursos hídricos do subsolo, pois, uma vez retirado das camadas superficiais, onde há maior teor de MO e atividade microbiana, a sua persistência no ambiente pode ser intensamente prolongada (Sarmah et al., 1998; Costa et al., 2000; Prata et al., 2001). Matallo et al. (2003), em estudos de lixiviação dos herbicidas diuron e tebuthiuron, concluíram que os dois herbicidas lixiviaram através da camada de $50 \mathrm{~cm}$, e o teor de matéria orgânica dos solos influenciou a capacidade de lixiviação desses herbicidas. Na Geórgia (EUA), foi detectado hexazinone tanto na água de escoamento superficial quanto no fluxo descendente de água no solo por vários meses após a aplicação de $1,62 \mathrm{~kg} \mathrm{ha}^{-1}$ de sua dosagem (Bouchard et al., 1988).

O herbicida imazapyr com $80 \mathrm{~mm}$ de chuva apresentou fitotoxidade no bioindicador até $25 \mathrm{~cm}$ de profundidade, ou seja, à medida que o índice pluviométrico aumentou, a profundidade de ação também aumentou. A atividade biológica e o seu movimento no solo são fortemente influenciados por fatores do solo, como textura, argila, umidade e $\mathrm{pH}$, que afetam diretamente a adsorção e, conseqüentemente, a mobilidade do imazapyr no perfil do solo (Michael \& Neary, 1993; Ismail \& Ahmad, 1994).

Wehtje et al. (1987) observaram maior mobilidade do imazapyr em solos argilosos, a qual diminuiu com a redução da umidade e do $\mathrm{pH}$ do solo. Entretanto, outros resultados foram observados por Vizantinopoulos \& Lolos (1994), que encontraram maior mobilidade no solo 

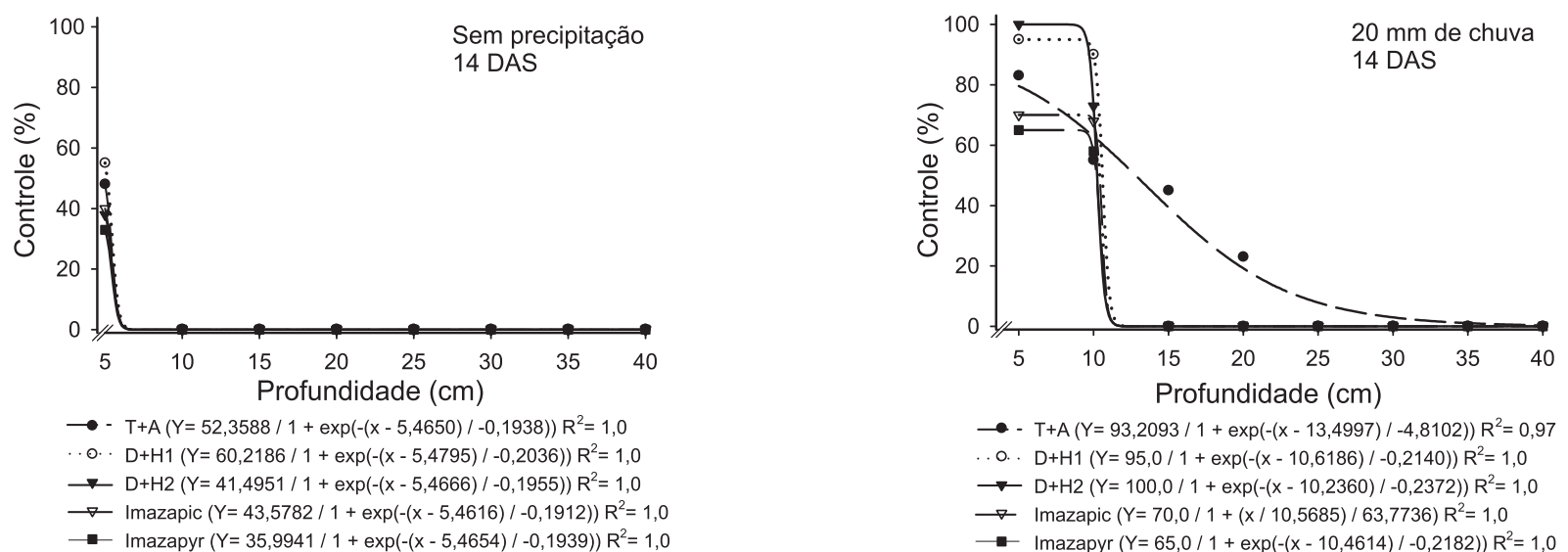

$\rightarrow-T+A(Y=93,2093 / 1+\exp (-(x-13,4997) /-4,8102)) R^{2}=0,97$

. o. . $\mathrm{D}+\mathrm{H} 1(\mathrm{Y}=95,0 / 1+\exp (-(\mathrm{X}-10,6186) /-0,2140)) \mathrm{R}^{2}=1,0$

$\rightarrow \mathrm{D}+\mathrm{H} 2(\mathrm{Y}=100,0 / 1+\exp (-(\mathrm{x}-10,2360) /-0,2372)) \mathrm{R}^{2}=1,0$

$\rightarrow$ Imazapic $(Y=70,0 / 1+(x / 10,5685) / 63,7736) R^{2}=1,0$

- Imazapyr $(Y=65,0 / 1+\exp (-(\mathrm{x}-10,4614) /-0,2182)) \mathrm{R}^{2}=1,0$
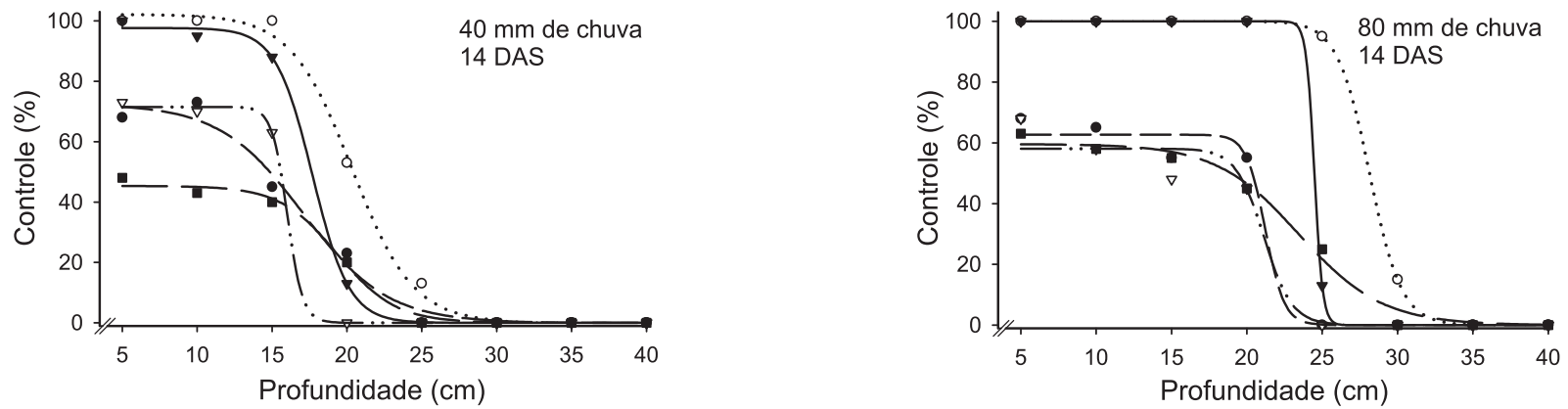

$\rightarrow-\mathrm{T}+\mathrm{A}(\mathrm{Y}=72,4403 / 1+\exp (-(\mathrm{x}-17,0980) /-2,8031)) \mathrm{R}^{2}=0,99$
$\cdots \mathrm{O} \cdot \mathrm{D}+\mathrm{H} 1(\mathrm{Y}=102,1113 / 1+\exp (-(\mathrm{x}-20,3447) /-2,0387)) \mathrm{R}^{2}=0,99$
$\rightarrow \mathrm{D}+\mathrm{H} 2(\mathrm{Y}=97,6354 / 1+\exp (-(\mathrm{x}-17,7027) /-1,2278)) \mathrm{R}^{2}=0,99$
$\rightarrow \quad$ Imazapic $(\mathrm{Y}=71,5004 / 1+\exp (-(\mathrm{x}-16,0302) /-0,5144))) \mathrm{R}^{2}=0,99$
$\rightarrow-$ Imazapyr $(\mathrm{Y}=45,3436 / 1+\exp (-(\mathrm{x}-19,3821) /-1,9367)) \mathrm{R}^{2}=0,99$

$\rightarrow-T+A(Y=62,6706 / 1+\exp (-(x-21,2396) /-0,6298)) R^{2}=0,99$ .0. $\mathrm{D}+\mathrm{H} 1(\mathrm{Y}=100,0109 / 1+\exp (-(\mathrm{x}-28,1458) /-1,0687)) \mathrm{R}^{2}=0,99$ $\rightarrow \mathrm{D}+\mathrm{H} 2(\mathrm{Y}=100,0 / 1+\exp (-(\mathrm{x}-24,4803) /-0,2734)) \mathrm{R}^{2}=1,0$ $\rightarrow$ Imazapic $(Y=58,0722 / 1+\exp (-(x-21,1388) /-0,9342)) R^{2}=0,97$ - Imazapyr $(Y=59,6070 / 1+\exp (-(x-23,4783) /-2,7550)) R^{2}=0,99$

Figura 1 - Lixiviação dos herbicidas trifloxysulfuron-sodium + ametryn (T+A), diuron + hexazinone (D+H) $(1-1.170+330 \mathrm{~g}$ ha-1 e $\left.2-1.330+160 \mathrm{~g} \mathrm{ha}^{-1}\right)$, imazapic e imazapyr, aplicados em colunas de solo e submetidos a diferentes precipitações $(0,20,40$ e $80 \mathrm{~mm}$ ), aos 14 dias após a semeadura da planta indicadora (Cucumis sativus).

com menor teor de argila. Essa diferença de mobilidade pode estar relacionada a fatores que estariam agindo sobre os fenômenos adsortivos, como as características mineralógicas e, ainda, o teor e tipo de matéria orgânica dos solos.

Pusino et al. (1997) constataram que a adsorção do imazapyr foi fortemente influenciada pelo $\mathrm{pH}$ e pela carga do componente adsorvente e que a presença de óxidos de ferro amorfo e de matéria orgânica e o $\mathrm{pH}$ do solo menor que 5 foram efetivos na sua retenção.

Os herbicidas trifloxysulfurom-sodium + ametryn e imazapic provocaram fitotoxicidade até $25 \mathrm{~cm}$ de profundidade. Em trabalhos anteriores, simulações de chuva de 20 e $40 \mathrm{~mm}$ provocaram lixiviação de trifloxysulfuron-sodium + ametryn até $15 \mathrm{~cm}$ de profundidade do solo. Já com $80 \mathrm{~mm}$ de chuva ocorreu distribuição do produto até a profundidade de $20 \mathrm{~cm}$, sendo considerada uma mistura herbicida que possui mobilidade no solo e potencial de lixiviação (Reis et al., 2006).

Concluiu-se que os herbicidas trifloxysulfurom-sodium + ametryn, imazapic, diuron + hexazinone (formulações 1 e 2) e imazapyr apresentaram tendência a serem lixiviados por influência das precipitações pluviais ou de irrigações artificiais, cuja movimentação no perfil do solo pode determinar a seletividade e/ou a eficiência no controle de plantas daninhas, assim como o potencial de contaminação de águas subterrâneas. 

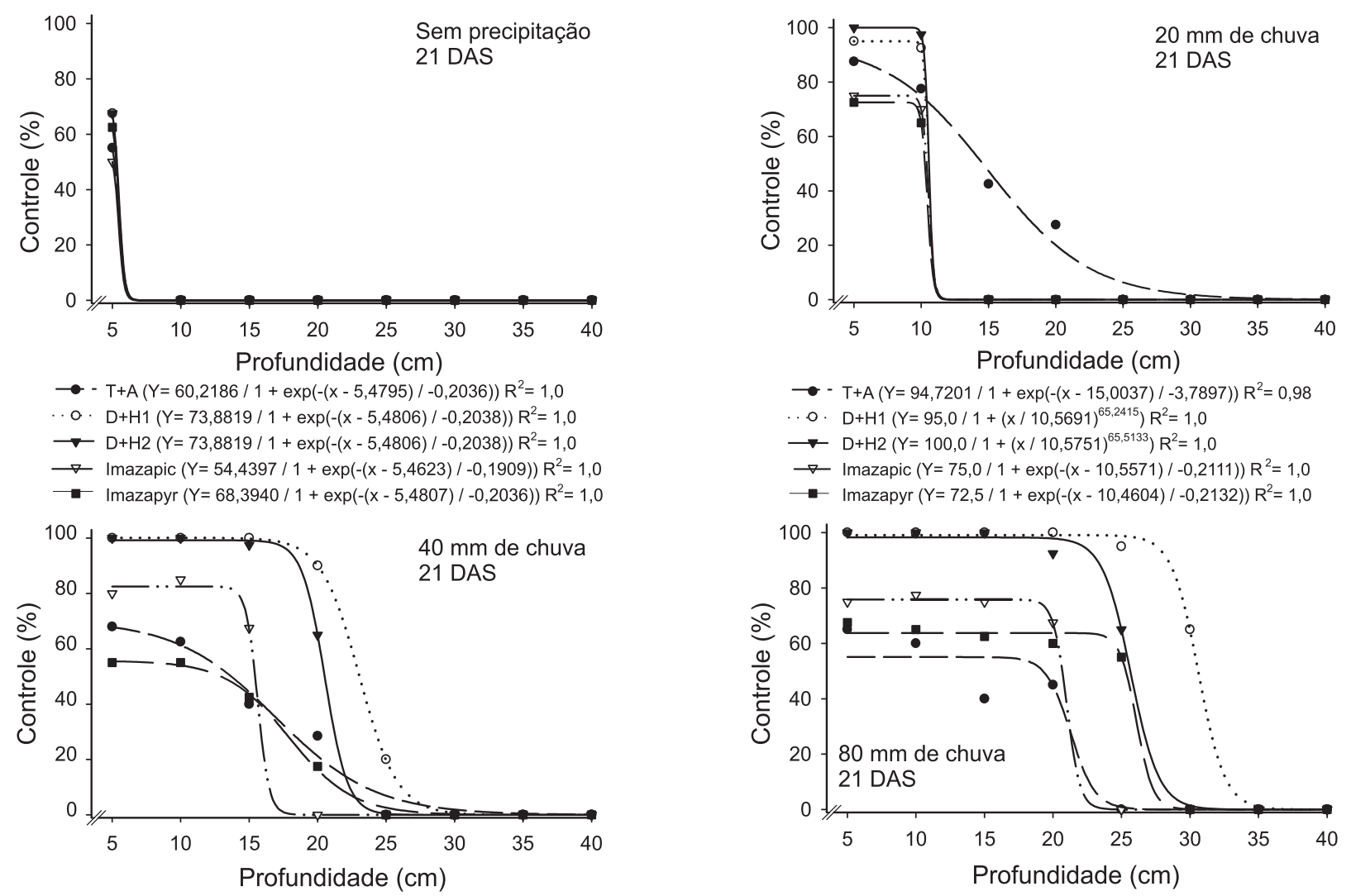

$\rightarrow-T+A(Y=70,2597 / 1+\exp (-(x-16,9562) /-3,6225)) R^{2}=0,98$

a. $\mathrm{D}+\mathrm{H} 1(\mathrm{Y}=100,0878 / 1+\exp (-(\mathrm{X}-23,0607) /-1,3939)) \mathrm{R}^{2}=0,99$

$\rightarrow D+H 2(Y=99,2116 / 1+\exp (-(x-20,5299) /-0,8266)) R^{2}=0,99$

$\rightarrow$ Imazapic $(Y=82,4998 / 1+\exp (-(X-15,6349) /-0,4221)) R^{2}=0,99$

$\longrightarrow$ Imazapyr $(Y=55,6885 / 1+\exp (-(x-17,9502) /-2,3176)) R^{2}=0,99$

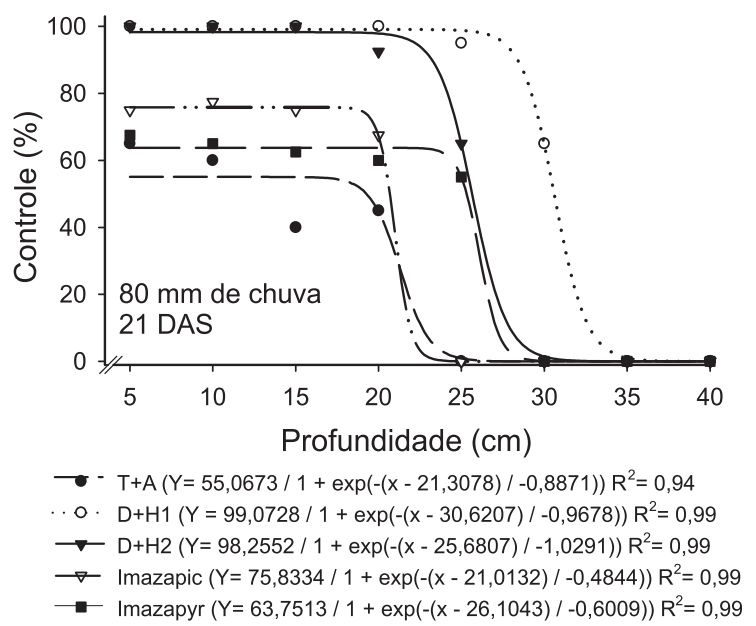

Figura 2 - Lixiviação dos herbicidas trifloxysulfuron-sodium + ametryn (T+A), diuron + hexazinone (D+H) $(1-1.170+330$ g ha-1 e $\left.2-1.330+160 \mathrm{~g} \mathrm{ha}^{-1}\right)$, imazapic e imazapyr, aplicados em colunas de solo e submetidos a diferentes precipitações $(0,20,40$ e $80 \mathrm{~mm}$ ), aos 21 dias após a semeadura da planta indicadora (Cucumis sativus).

\section{LITERATURA CITADA}

ASOCIATION LATINO AMERICANA DE MALEZAS ALAM. Recomendaciones sobre unificación de los sistemas de evaluación em ensayos de control de malezas. ALAM, v. 1, p. $35-38,1974$

BARIZON, R. R. M. et al. Simulação do transporte e da sorção de imazaquin em colunas de solo. R. Bras. Ci. Solo, v. 30, n. 4 , p. $615-623,2006$.

BOUCHARD, D. C.; LAVY, T. L.; LAWSON, E. R. Mobility and persistence of hexazinone in a forest watershed. J. Environ. Qual., v. 14, n. 2, p. 229-233, 1985.
BUHLER, D. D.; HARTZLER, R. G.; FORCELLA, F. Implications of weed seed bank dynamics to weed management. Weed Sci., v. 45, n. 3, p. 329-336, 1997.

CARTER, A. D. Herbicide movement in soils: principles, pathways and processes. Weed Res., v. 40, n. 1, p. 113-122, 2000 .

COSTA, E. A. D.; GELMINI, G. A.; ZAMBON, S. Avaliação de isoxaflutole aplicado isoladamente ou em mistura de tanque no nivelamento do solo para controle préemergente de infestantes em cana-planta. In: CONGRESSO BRASILEIRO DA CIÊNCIA DAS PLANTAS DANINHAS, 22., 2000, Foz do Iguaçu. Resumos... Londrina: SBCPD, 2000. p. 295. 
EMPRESA BRASILEIRA DE PESQUISA

AGROPECUÁRIA - EMBRAPA. Sistema brasileiro de classificação de solos. Brasília: 1999. $412 \mathrm{p}$

INOUE, M. H. et al. Avaliação do potencial de lixiviação de imazapic e isoxaflutole em colunas de solo. In: CONGRESSO BRASILEIRO DA CIÊNCIA DAS PLANTAS DANINHAS, 25., 2006, Brasília. Resumos... Brasília: SBCPD; UNB; Embrapa Cerrados, 2006. p. 131.

ISMAIL, B. S.; AHMAD, A. R. Attenuation of the herbicidal activities of glufosinate -ammonium and imazapyr in two soils. Agric. Ecosyst. Environ., v. 47, p. 279-285, 1994.

LAABS, V. et al. Pesticides in surface water, sediment and rainfall of the northeastern Pantanal basin, Brasil.

J. Environ. Qual., v. 31, n. 3, p. 1636-1648, 2002.

MATALLO, M. B. et al. Lixiviação dos herbicidas tebuthiuron e diuron em colunas de solo. Pesticidas.

R. Ecotoxicol. Meio Amb., v. 13, p. 83-90, 2003.

MICHAEL, J. L.; NEARY, D. G. Herbicide dissipation studies in southern forest ecosystems. Environ. toxicol. Chem., v. 12, p. 405-410, 1993.

MITCHELL, C. et al. Sediments, nutrients and pesticide residues in event flow conditions in streams of the Mackay Whitsunday region, Australia. Marine Poll. B., v. 51, p. 23$36,2005$.

PASQUALETTO, A. et al. Avaliação de lixiviado de imidazolinona por meio de bioensaio. Pesq. Agropec. Trop., v. 29, n. 1, p. 1-5, 1999.

PESSOA, M. C. P. Y. et al. Simulação de herbicidas utilizados no monocultivo de cana-de-açúcar em Latossolos da área de recarga do Aqüífero Botucatu (Guarani) em Ribeirão Preto, SP. R. Ci. Rural, v. 4, n. 1, p. 15-24, 1999.

PFEUFFER, R. J.; RAND, G. M. South Florida ambient pesticide monitoring program. Ecotoxicology, v. 13, n. 3, p. 195-205, 2004.

PRATA, F. et al. Degradação e sorção de ametrina em dois solos com aplicação de vinhaça. Pesq. Agropec. Bras., v. 36, n. 7, p. 975-981, 2001.

PRATA, F. et al. Glyphosate sorption and desorption in soils with different phosphorous levels. Sci. Agric., v. 60, n. 1, p. 175-180, 2003.
PROCÓPIO, S. D. et al. Efeito da irrigação inicial na profundidade de lixiviação do herbicida s-metolachlor em diferentes tipos de solo. Planta Daninha, v. 19, n. 3, p. 409-417, 2001.

PUSINO, A.; PETRETTO, S.; GESS A, C. Adsorption and desorption of imazapyr by soil. J. Agric. Food Chem., v. 49, n. 3, p. 1012-1016, 1997.

REIS, M. R. et al. Influência da chuva na lixiviação do herbicida ametryn + trifloxysulfuron-sodium. In: CONGRESSO BRASILEIRO DA CIÊNCIA DAS PLANTAS DANINHAS, 25., 2006, Brasília. Resumos... Brasília: SBCPD; UNB; Embrapa Cerrados, 2006. p. 151.

ROSSI, C. V. S.; ALVES, P. L. C. A.; MARQUES JUNIOR, J. Mobilidade do sulfentrazone em Latossolo Vermelho e em Chernossolo. Planta Daninha, v. 23, n. 4, p. 701-710, 2005.

SARMAH, A.; KOOKANA, R.; ALSTON, A. Fate and behaviour of triasulfuron, metsulfuron-methyl and chlorsulfuron in the Australian soil environment: A review. J. Agric. Res., v. 49, n.5, p. 775-790, 1998.

SOUTHWICK, L. M. et al. Potential influence of sugarcane cultivation on estuarine water quality of Louisiana's gulf coast. J. Agric. Food Chem., v. 50, n. 15, p. 4393-4399, 2002 .

TANABE, A. et al. Seasonal and special studies on pesticide residues in surface waters of the Shinano river in Japan. $\mathbf{J}$. Agric. Food Chem., v. 49, n. 7, p. 3847-3852, 2001.

VELINI, E. D. Comportamento de herbicidas no solo. In: SIMPÓSIO NACIONAL SOBRE MANEJO DE PLANTAS DANINHAS EM HORTALIÇAS, 1992, Botucatu. Resumos... Botucatu: 1992. p. 44-64.

VIVIAN, R. et al. Persistência e lixiviação de ametryn e trifloxysulfuron-sodium em solo cultivado com cana-deaçúcar. Planta Daninha, v. 25, n. 1, p. 111-124, 2007.

VIZANTINOPOULOS, S.; LOLOS, P. Persistence and leaching of the herbicide imazapyr in soil. Bull. Environ Contam. Toxicol., v. 52, p. 404-410, 1994.

WEHTJE, G. et al. Sorption and mobility of sulfometuron and imazapyr in five Alabama soils. Weed Sci., v. 35, n. 6, p. 858-864, 1987. 